\title{
Actitud de técnicos paramédicos en un hospital de la Región Metropolitana frente al paciente VIH/SIDA
}

\author{
XIMENA FERRER(1), ANGÉLICA CAZENAVE(1), \\ CONSTANZA AGLIATI(1), y MARIELA GORIGOITÍA(1)
}

\section{RESUMEN}

Estudio cuantitativo y transversal, que pretende conocer la actitud, entendida como conocimientos, sentimientos y conductas, de técnicos paramédicos frente a la persona VIH/SIDA. La muestra queda constituida por 77 técnicos paramédicos que se desempeñan en el servicio de medicina y en el servicio de urgencia de un Hospital de la Región Metropolitana. Para la obtención de la información se aplica la escala autoaplicada de Stewart, V. Castellano. A., validada con un indice de alpha de Crombach de 0,74 por la Escuela de Enfermería de la Pontificia Universidad Católica de Chile. Los resultados muestran que el $85 \%$ de los técnicos paramédicos son mujeres y el 65\% tiene entre 30 y 45 años. El estudio muestra, con respecto al componente afectivo de la actitud, que un 35,1\% tiene un sentimiento indiferente y un 55,8\% un sentimiento negativo hacia las personas VIH/SIDA. En cuanto al nivel de conocimiento, sólo un $47,9 \%$ posee los adecuados y un 50,6\% tiene un comportamiento indiferente. En cuanto a la actitud general frente al paciente VIH/SIDA, un 45,5\% presenta una actitud indiferente. Esta sugiere un gran desafío para los equipos de salud, no sólo desde el punto de vista técnico sino desde el aspecto ético y la atención de calidad que se pretende dar a las personas con VIH/ SIDA. Como plantea Eisenberg, es muy dificil el cambio de las actitudes negativas, sin embargo, es posible modificar las indiferentes, para lo que se requiere no sólo de información, sino también formar a los técnicos paramédicos y al equipo de salud en general, en habilidades específicas que les permitan enfrentar mejor sus propias dificultades con algunos pacientes y mejorar la atención que brindan, desde una perspectiva mas humana.

Palabras claves: VIH/SIDA, técnicos paramédicos, actitud.

\section{ABSTRACT}

\section{ATTITUDE OF PARAMEDIC TECHNICIANS FROM A HOSPITAL OF} THE METROPOLITAN REGION TOWARD VIH/AIDS PATIENTS

This is a quantitative and transversal study which seeks to find out the attitude, understood as knowledge, feelings about and conduct toward HIV/AIDS patients shown by paramedic technicians. The sample consists of 77 paramedic technicians working in the medical and emergency services of a hospital in the Metropolitan Region. For the purpose of obtaining the information, the autoapplied scale of V. Stuart and A. Castellano was used, validated with a Crombach alpha

(1) Escuela de Enfermería. Facultad de Medicina. Pontificia Universidad Católica de Chile. xferrer@puc cl

Financiamiento: Proyecto Aids International Traning and Research Program (AITRP) 
index of 0.74 by the Nursing School of the Pontificial Catholic University of Chile. The results of the study show that $85 \%$ of the paramedic technicians are women, and that $65 \%$ are between 30 and 45 years old. With respect to the affective component of the attitude, the study shows that $35.1 \%$ of those interviewed had indifferent feelings, and $55.8 \%$ had negative feelings toward HIVI AIDS patients. As far as the level of knowledge is concerned, only $47.9 \%$ is adequately informed, and $50.6 \%$ shows an indifferent attitude. The indifferent attitude presents a great challenge to health teams, not only from the technical, but also from the ethical point of view and the quality of health care being attempted to be provided to HIV/AIDS patients. As Eisenberg states, it is very difficult to change negative attitudes, nevertheless, it is possible to modify those that are indifferent, a purpose for which one requires not only information, but also the capacity of forming technical paramedics and the health team in general in specific abilities which will allow them to face their own difficulties with some patients more adequately, and to improve the attention they offer by viewing it from a more humane perspective.

Key words: HIV/AIDS, paramedical technicians, attitude.

\section{INTRODUCCIÓN}

El VIH/SIDA tiene consecuencias sociales y psicológicas entre los trabajadores de la salud que atienden a las personas con esta enfermedad. La obligatoriedad de trabajar con enfermos VIH (+) se acompaña de miedo al contagio (en ocasiones irracional), incomodidad por las dimensiones sexuales de la enfermedad y sensación de indefensión puesto que no existe tratamiento efectivo. Probablemente, debido a esto, es que algunos profesionales de la salud evitan el cuidado de los enfermos con VIH/ SIDA; en cambio otros, asumen su trabajo pero pueden terminar emocionalmente exhaustos y "quemados" por la presión que suponel.

El estudio de $\mathrm{Kat}^{2}$ realiza una comparación entre las percepciones referidas a diferentes enfermedades crónicas, entre ellas el VIH/ SIDA. Dicha investigación revela que los enfermos de este mal, son los más negativamente evaluados, ya que son vistos como los responsables de su enfermedad. Holland ${ }^{1}$, plantea que el enfermo con VIH/SIDA es recibido con recelo por los profesionales de la salud y los auxiliares paramédicos. Este rechazo, a su vez, es percibido claramente por el enfermo, aumentando así su aislamiento o produciéndose recíprocamente rechazo hacia todo intento terapéutico.

El personal que asiste a estos enfermos debe ser capaz de aceptar y entender estilos de vida sociales y sexuales distintos. No deben tomar decisiones por los pacientes, sino ayudarles a tomar las suyas propias. Por ello es importante que estén familiarizados con los avances en la enfermedad, opciones de tratamiento, ensayos clínicos y marcadores de pronóstico. Además, es necesario escuchar al paciente con atención, sin darle la sensación de que se tiene prisa en concluir la visita ${ }^{3}$. Encuestas realizadas a trabajadores de la salud revelan que aquellos que están más informados tienen menos miedo al contagio $\mathrm{y}$, por lo tanto, menor rechazo a estos pacientes ${ }^{4}$.

Enfermería es parte de este equipo y debe estar preparado para brindar un ambiente de apoyo y cuidado, alimentación, control y manejo de síntomas a través de una relación que permita comprender y dar respuesta a sus necesidades reales. El personal de enfermería debe brindar un ambiente de aceptación al paciente, es decir, debe dejar de lado los prejuicios o sentimientos personales acerca del estilo de vida de éste ${ }^{5}$. La actitud del personal de la salud ante el enfermo de VIH/SIDA es fundamental para obtener su confianza.

$\mathrm{Kat}^{2}$ define la actitud como la integración de los componentes cognitivo, afectivo y conductual. En tanto, las actitudes son consideradas como mediadores entre un estímulo y un comportamiento o respuesta, son también un proceso cognitivo que incluye la selección, codificación e interpretación de 
la información proveniente del estímulo ${ }^{3}$. El componente cognitivo es la categoría conceptual de objetos o sucesos a los que se dirige la actitud. Este componente define al objeto de la actitud, especificando personas o eventos a los que la actitud es dirigida ${ }^{6}$.

El componente afectivo se define como el sentimiento a favor o en contra de un determinado objeto social, y también como el más enraizado en la persona y el que más se resiste al cambio ${ }^{6}$. El elemento afectivo incluye los sentimientos y emociones que acompañan a una creencia o idea nuclear, en tanto interactúan los componentes cognitivos y afectivos, dan la posibilidad de ir conformando lo que el término actitud significa ${ }^{6}$.

El componente conductual es el resultado de la interacción entre los componentes cognitivo y afectivo. Ambos tienden a cierta congruencia, así los sentimientos positivos tienden a generar disposiciones en las personas para entablar un contacto más estrecho y una experiencia prolongada con el objeto de la actitud $^{6}$. Por otra parte, al estudiar la actitud frente a diferentes eventos, es importante considerar que es más factible cambiar los comportamientos indiferentes que los negativos.

La ayuda a estos pacientes debe estar orientada a maximizar su calidad de vida y bienestar social y ayudarles a enfrentarse a la enfermedad. La actitud, es decir, conocimiento, sentimientos y conducta, tiene relación con la forma en que se percibe la imagen de uno mismo, de los demás y de los acontecimientos que nos rodean, lo cual va a determinar la manera cómo nos comportamos. Está vinculada, a su vez, con los esquemas mentales, los que se han estructurado en la infancia y que continúan reforzándose en la vida diaria. La actitud implica hábitos de pensamiento que condicionan las reacciones y la conducta con respecto a un objeto, persona, hecho o situación, éstas son aprendidas y, por lo tanto, modificables.

Es importante considerar que cada miembro del equipo de salud tiene sus propios valores, actitudes y creencias, y cada día se ven enfrentados a personas que pueden ser considerablemente diferentes en estos aspectos. A esto se le suma el prejuicio que el VIH/SIDA evoca, que trae consigo estigma, discriminación, hostilidad y opresión, y podría, incluso, aplastar los valores que son el fundamento del cuidado humano del enfermo?. De hecho, se ha visto que las actitudes y percepciones hacia el VIH/ SIDA influyen de manera decisiva en la forma en que los sujetos y las instituciones sociales responden 8 .

\section{MATERIAL Y MÉTODO}

El propósito de este estudio es conocer las actitudes (conocimientos, sentimientos y conductas) del personal de enfermería no profesional frente a los enfermos VIH/SIDA en un hospital de la Región Metropolitana. El objetivo general es describir las actitudes que tiene el personal técnico paramédico frente al paciente con VIH/SIDA.

Es un estudio de tipo descriptivo y transversal, realizado en el personal técnico paramédico que realiza atención directa con el paciente, en los servicios de urgencia y medicina de un Hospital de la Región Metropolitana.

El universo corresponde a 84 técnicos paramédicos y la muestra queda constituida por 77 técnicos, que aceptan participar en el estudio mediante el consentimiento informado. La obtención de la información se realiza a través de la escala de actitudes, de autoaplicación, modificada por Stewart V (2001), basada en la escala de Castellano, $\mathrm{A}^{10}$, validada por el proyecto Aids International Research Program (AITRP) de la Escuela de Enfermería de la Pontificia Universidad Católica de Chile con un coeficiente de alpha de Cronbach de 0,74.

\section{RESULTADOS}

Los resultados del estudio muestran que el $85,7 \%$ de las personas encuestadas corresponde al sexo femenino, lo que coincide con lo observado en los distintos servicios de salud pública del país y el $65 \%$ de los técnicos paramédicos tienen entre 30 y 45 años. (Tabla 1 ).

Al observar la información con respecto al componente afectivo de la actitud, se manifiesta que un $35,1 \%$ de la muestra presenta un sentimiento indiferente hacia las personas VIH/ 
SIDA, y un 55,8\% un sentimiento negativo. En el rango de edad entre 30 y 45 años, casi un $30 \%$ tiene sentimiento indiferente y un $27,2 \%$ tiene sentimiento negativo, en cambio en los grupos de menores de 30 años y mayores de 45 años se concentra la actitud en un sentimiento negativo.

Esto es importante, ya que modificar la actitud tiene mayores posibilidades cuando ésta es indiferente, para lo cual -probablemente- es necesario escuchar a los técnicos paramédicos para saber a qué sentimientos en concreto se refieren, saber qué sienten frente a las personas VIH/SIDA y ampliar la información respecto a la enfermedad. Llama la atención que los grupos extremos en edad, sean quienes muestran mayor sentimiento negativo, lo que podría estar explicado en los jóvenes por desconocimiento del comportamiento de las personas con VIH/
SIDA al tener poca experiencia, y en el grupo de mayor edad por la dificultad en cambiar ideas y prejuicios respecto a esta enfermedad. (Tabla 2).

En cuanto a nivel de conocimiento que tienen los técnicos paramédicos sobre VIH/ SIDA, se observa que menos de la mitad de la muestra $(47,9 \%)$ posee conocimiento adecuado. Esto indica la importancia de entregar información y educación sobre las personas con VIH/SIDA y sobre la enfermedad en sí misma. La posibilidad de cambiar la conducta está ligada fuertemente al ámbito cognitivo y su relación con el ámbito afectivo. En este estudio se observa como ambos componentes (cognitivo y afectivo) están en déficit para lo esperado y por lo tanto para realizar un cuidado de calidad. Al comparar el grado de conocimiento con la edad de los encuestados no se encuentran

Tabla 1. Sentimiento frente al paciente VIH/SIDA de los técnicos paramédicos según rango de edad

\begin{tabular}{|c|c|c|c|c|c|c|c|c|}
\hline \multirow{3}{*}{ Edad } & \multicolumn{8}{|c|}{ Sentimiento } \\
\hline & \multicolumn{2}{|c|}{ Positivo } & \multicolumn{2}{|c|}{ Indiferente } & \multicolumn{2}{|c|}{ Negativo } & \multicolumn{2}{|c|}{ Total } \\
\hline & $\mathbf{n}$ & $\%$ & n & $\%$ & $\mathbf{n}$ & $\%$ & $\mathbf{n}$ & $\%$ \\
\hline$<30$ & 1 & 1,3 & 3 & 3,9 & 11 & 14,3 & 15 & 19,5 \\
\hline $30-45$ & 6 & 7,9 & 23 & 29,8 & 21 & 27,2 & 50 & 64,9 \\
\hline$>45$ & - & - & 1 & 1,3 & 11 & 14,3 & 12 & 15,6 \\
\hline Total & 7 & 9,2 & 27 & 35,0 & 43 & 55,8 & 77 & 100,0 \\
\hline
\end{tabular}

Tabla 2. Conocimiento de los técnicos paramédicos según edad

\begin{tabular}{|c|c|c|c|c|c|c|c|c|}
\hline \multirow{3}{*}{ Edad } & \multicolumn{8}{|c|}{ Conocimiento } \\
\hline & \multicolumn{2}{|c|}{ Adecuado } & \multicolumn{2}{|c|}{ Regular } & \multicolumn{2}{|c|}{ Inadecuado } & \multicolumn{2}{|c|}{ Total } \\
\hline & $\mathbf{n}$ & $\%$ & $\mathbf{n}$ & $\%$ & $\mathbf{n}$ & $\%$ & $\mathbf{n}$ & $\%$ \\
\hline$<30$ & 9 & 11,7 & 3 & 3,9 & 3 & 3,9 & 15 & 19,5 \\
\hline $30-45$ & 24 & 31,0 & 14 & 18,2 & 12 & 15,7 & 50 & 64,9 \\
\hline$>45$ & 4 & 5,2 & 6 & 7,9 & 2 & 2,5 & 12 & 15,6 \\
\hline Total & 37 & 47.9 & 23 & 30,0 & 17 & 22,1 & 77 & 100,0 \\
\hline
\end{tabular}

Tabla 3. Comportamiento hacia el paciente VIH/SIDA de los técnicos paramédicos según edad

\begin{tabular}{|c|c|c|c|c|c|c|c|c|}
\hline \multirow{3}{*}{ Edad } & \multicolumn{8}{|c|}{ Comportamiento } \\
\hline & \multicolumn{2}{|c|}{ Positivo } & \multicolumn{2}{|c|}{ Indiferente } & \multicolumn{2}{|c|}{ Negativo } & \multicolumn{2}{|c|}{ Total } \\
\hline & n & $\%$ & $n$ & $\%$ & n & $\%$ & $n$ & $\%$ \\
\hline$<30$ & 1 & 1,3 & 7 & 9,1 & 7 & 9,1 & 15 & 19.5 \\
\hline $30-45$ & 8 & 10,4 & 30 & 38,9 & 12 & 15,6 & 50 & 64,9 \\
\hline$>45$ & 4 & 5,2 & 2 & 2,6 & 6 & 7,8 & 12 & 15,6 \\
\hline Total & 13 & 16,9 & 39 & 50,6 & 25 & 32,5 & 77 & 100,0 \\
\hline
\end{tabular}


Tabla 4. Actitud hacia el paciente VIH/SIDA de los técnicos paramédicos según edad

\begin{tabular}{|c|c|c|c|c|c|c|c|c|}
\hline \multirow{3}{*}{ Edad } & \multicolumn{8}{|c|}{ Actitud } \\
\hline & \multicolumn{2}{|c|}{ Positivo } & \multicolumn{2}{|c|}{ Indiferente } & \multicolumn{2}{|c|}{ Negativo } & \multicolumn{2}{|c|}{ Total } \\
\hline & $\mathrm{n}$ & $\%$ & $\mathbf{n}$ & $\%$ & $\mathbf{n}$ & $\%$ & $\mathbf{n}$ & $\%$ \\
\hline$<30$ & 1 & 1,3 & 4 & 5,2 & 10 & 12,9 & 15 & 19,5 \\
\hline $30-45$ & 12 & 15,6 & 25 & 32,5 & 13 & 16,9 & 50 & 64,9 \\
\hline$>45$ & - & - & 6 & 7,8 & 6 & 7,8 & 12 & 15,6 \\
\hline Total & 13 & 16,9 & 35 & 45,5 & 29 & 37,6 & 77 & 100,0 \\
\hline
\end{tabular}

diferencias significativas. (Tabla 3).

Respecto al comportamiento que presentan los técnicos paramédicos, se observa que la mitad $(50,6 \%)$ tiene un comportamiento indiferente y más o menos un tercio de la muestra presenta en el ámbito de la conducta una actitud negativa $(32,5 \%)$, lo que puede perjudicar directamente a las personas que son atendidas por ellos, sobre todo si no toman conciencia de tener esta actitud deficitaria hacia estos pacientes, ya que la persona que reconoce esta conducta negativa puede rechazar el cuidado y agravar su propio estado.

Al comparar por edad, el grupo entre $30 \mathrm{y}$ 45 años presenta en su mayoría un comportamiento indiferente. Al observar ambos extremos de edad se encuentra un comportamiento mayoritariamente negativo, lo que se relaciona con los sentimientos que presentan estos grupos frente a los pacientes con VIH/ SIDA. (Tabla 4).

En cuanto a la actitud general frente al paciente VIH/SIDA, el mayor porcentaje se concentra en la categoría indiferente con un $45,5 \%$. La actitud indiferente sugiere un gran desafío, tal como plantean Rosemberg y Hovland ${ }^{1}$ existe dificultad en el cambio de actitudes negativas, sin embargo, es posible cambiar las actitudes indiferentes y reforzar las positivas.

Es importante destacar que un tercio de los técnicos paramédicos muestra una actitud general negativa $(37,6 \%)$. Se observa del total del grupo entre 30 y 45 años, la mitad muestra una actitud indiferente. Se destaca la actitud negativa en los grupos etarios menores de 30 años y mayores de 45 años, lo que se relaciona con el sentimiento y comportamiento negativo en estos grupos de edad.

\section{DISCUSIÓN}

Ya han transcurrido casi veinte años desde la aparición del primer caso de SIDA en nuestro país, y aún hoy se observa que trabajadores de la salud tienen desconocimiento de la enfermedad y no saben como enfrentarse a las personas con VIH/SIDA en este hospital de la Región Metroplitana.

Al observar los conocimientos de los técnicos paramédicos sobre VIH/SIDA, más de la mitad presenta un conocimiento entre regular e inadecuado lo que es relevante de considerar, ya que, quienes saben más pueden tener menos miedo al contagio y presentar menor rechazo a los pacientes. Por otra parte, con relación a la conducta y los sentimientos de los técnicos paramédicos, un número importante presentan comportamiento y sentimiento negativo, lo que influye directamente en el cuidado de los pacientes.

Diferentes estudios 2.89 .11 , demuestran una actitud discriminatoria y negativa hacia las personas con VIH/SIDA, lo que concuerda con los resultados obtenidos en esta investigación, observandose una actitud negativa en más de un tercio de la muestra $(37,6 \%)$ y una actitud indiferente en un $45,5 \%$.

Llama la atención que aún cuando el tema del VIH/SIDA es tratado en la formación y capacitación de los trabajadores de la salud y, es un tema tratado a nivel público, con diferentes programas y campañas, persiste en los técnicos paramédicos de este centro de salud una actitud que puede ser discriminatoria $\mathrm{y}$ donde los sentimientos juegan un papel relevante.

Estos resultados implican un desafío para el hospital y el personal de enfermería donde 
se realizó el estudio, se hace necesario la creación e implementación de programas educativos con el fin de lograr modificar actitudes indiferentes, e intentar comprender a que se refiere esa indiferencia para estas personas, ya que podría significar negación, poco compromiso o incluso discriminación. La capacitación de los técnicos, por lo tanto, debe incluir no sólo los aspectos técnicos del cuidado sino también los aspectos éticos y relacionales de la atención y ayuda a las personas enfermas.

\section{REFERENCIAS}

1.- HOLLAND J C, JACOBSEN P, BRIEBART $W$. Aspectos psiquiátricos y psicológicos del VIH, 1992; ( $3^{\mathrm{a}}$ Edición) Philadelphia: Editorial Lippincot.

2.- KATZ I, HASS G, PARISI, N, ASTONE J, MC EVADDY D. Lay people's and health care personnel's perception of cancer, aids, cardiac, and Diabetic patients. Psychological Reports 1987; 60: 615-29.

3.- ROVIRA M D, URIZ E, RODRÍGUEZ C. Comportamiento y actitud de los profesionales de enfermería hospitalaria ante los pacientes VIH positivo. Revista SEISIDA 1994; 1 (5): 513-7.

4.- SASTRE J, USIETO R. Factores psicosociales en el SIDA. Apoyo social. Revista SEISIDA 1993; 1 (4): 5-8.

5.- SEPÚlVEDA C. SIDA 1997. (2ªdición) Santiago Chile: Editorial Mediterráneo.

6.- EISENBERG G FANY. Las actitudes. Revista de Investigación al Día 2000; 1 (6).

7.- OMS. Valores y actitudes en el asesoramiento acerca del HIV. Programa global sobre SIDA. Manual de preparación. Tm/7. 1997.

8. VIDAL, F. Factores que influyen en las actitudes públicas hacia el SIDA, 1995. Tesis Magíster en Sociología. Santiago, Chile: Instituto de Sociología, Pontificia Universidad Católica de Chile.

9.- PEDRAZ M; NAVARRO A. Actitudes del personal de enfermería frente al paciente con SIDA. Revista Metas de Enfermería 1999; Nº Julio / Agosto.

10.- CASTELLANO A. Actitud de los profesionales de la salud de las dependencias docentes asistenciales de la PUC frente al SIDA, 1993; Tesis Magíster Salud Pública. Universidad de Chile.

11.- MARDONES V, REYES M. Factores Psicosociales que influyen en los comportamientos de rechazo hacia los pacientes con síndrome de inmunodeficiencia adquirida, 1991; Escuela de Psicología, Santiago, Chile: Pontificia Universidad Católica de Chile.

Usted puede comentar éste y otros artículos publicados en la Revista Chilena de Salud Pública, enviando un correo electrónico a revistasp@med.uchile.cl 